\title{
Modélisation De La Dynamique Spatio - Temporelle De L'occupation Du Sol Et Analyse Des Changements Du Territoire De La Lama Au Bénin
}

\author{
Dr. Tchibozo É. A. M., Maître de Conférences
}

Centre Universitaire d'Adjarra (CUA), Université d'Abomey - Calavi (Bénin), Faculté des sciences Humaines et Sociales (FASHS), Département de Géographie et Aménagement du Territoire, Laboratoire des Applications Géomatiques et Gestion de l'Environnement

Doi:10.19044/esj.2020.v16n6p499 ～URL:http://dx.doi.org/10.19044/esj.2020.v16n6p499

\section{Résumé}

Cette étude s'inscrit dans un contexte de territorialisation du développement agricole du Bénin. L’objectif est de modéliser la dynamique spatio-temporelle de l'occupation du sol et d'analyser les changements du territoire de la Lama d'ici aux horizons Bénin - Alafia 2025 et des Objectifs du Développement Durable (ODD) 2030. La méthode utilisée est une approche géographique et diachronique de l'étude de l'occupation sol à partir de la cartographie par classification supervisée d'image, de la modélisation prédictive et de l'analyse spatiale. Les résultats obtenus ont montré que la dynamique spatio-temporelle de l'occupation du sol est caractérisée par la pression anthropique sans cesse croissante de l'agriculture et de l'urbanisation sur les formations naturelles. Cette situation exprime une intensité de changement qui est relativement importante pour les savanes arborée et arbustive (78 \%) et la forêt claire et savane boisée (68\%). De 2000 à 2030, les changements lents de l'occupation du sol ont augmenté de 65,86\% et ceux rapides de 79,79\%. Les zones de stabilité ont diminué de 60,85\% et constituent une menace actuelle pour le secteur d'étude. L'analyse des niveaux de fragmentation des unités d'occupation du sol pendant les périodes d'étude a révélé que la forêt claire et savane boisée, les marécages et les eaux de surface vont connaître une fragmentation régressive alors que les savanes arborée et arbustive seront exposées à une fragmentation progressive. En 2030, cette dernière unité sera fragmentée à $99 \%$ à cause des changements qui y sont observés.

Mots clés : Modélisation, Dynamique De L’occupation Du Sol, Fragmentation, Territoire Du Lama, Bénin 


\title{
Modelling of Spatio-Temporal Dynamics of Land Use and Analysis of Changes in the Territory from Lama to Benin
}

\author{
Dr. Tchibozo É. A. M., Maître de Conférences \\ Centre Universitaire d'Adjarra (CUA), Université d'Abomey - Calavi \\ (Bénin), Faculté des sciences Humaines et Sociales (FASHS), Département \\ de Géographie et Aménagement du Territoire, Laboratoire des Applications \\ Géomatiques et Gestion de l'Environnement
}

\begin{abstract}
This study is part of a context of territorialization of Benin's agricultural development. The objective is to model the spatio-temporal dynamics of land use and to analyse the changes in the Lama's territory between now and the horizons Benin - Alafia 2025 and Sustainable Development Objectives (SDO) 2030. The method used is a geographical and diachronic approach to land use study based on mapping by supervised image classification, predictive modelling and spatial analysis. The results obtained showed that the spatio-temporal dynamics of land use are characterized by the ever-increasing anthropogenic pressure of agriculture and urbanization on natural formations. This situation expresses an intensity of change that is relatively high for tree and shrub savannah (78\%) and open forest and wooded savannah (68\%). From 2000 to 2030, slow changes in land use increased by $65.86 \%$ and rapid changes by $79.79 \%$. Areas of stability decreased by $60.85 \%$ and are a current threat to the study area. The analysis of the fragmentation levels of the land use units during the study periods revealed that open forest and wooded savannah, swamps and surface waters will experience regressive fragmentation while tree and shrub savannah will be exposed to progressive fragmentation. By 2030, the latter unit will be $99 \%$ fragmented because of the changes observed there.
\end{abstract}

Keywords: Modelling, Land Use Dynamics, Fragmentation, Lama Territory, Benin

\section{Introduction}

En Afrique occidentale, les ressources naturelles enregistrent des mutations perceptibles aussi bien à l'échelle locale que régionale. Les causes souvent évoquées sont la croissance de la population et la baisse des 
précipitations (FAO, 2015 ; Hibbarda et al., 2010 ; Lawrence et al., 2010). Aujourd'hui, l'ensemble des régions du monde est confronté à la détérioration des ressources naturelles, à la pression croissante sur les écosystèmes et à la perte de la diversité biologique (Brun et al., 2018 ; FAO-ODD, 2017).

Au Bénin, les ressources naturelles et la biodiversité servent encore de fondement à de nombreux secteurs importants, notamment l'agriculture, la pêche, l'élevage, l'alimentation en eau et les forêts. Leur dégradation s'est intensifiée ces dernières années à cause des pressions démographiques et une exploitation excessive du sol (Toko Mouhamadou, 2014 ; Mama et al., 2014 ; Oloukoi, 2013). Plusieurs rapports d'étude ont évalué les impacts régionaux de la dynamique de l'occupation du sol et de l'utilisation des terres sur les processus climatiques ou biophysiques et hydrologiques (Ministère du Cadre de Vie et du Développement Durable du Bénin, 2017 ; Ministère de l'Environnement, de l'Habitat et de l'Urbanisme, Direction Générale de l'Environnement, 2011 ; Takahashi et al., 2010). Si la plupart des recherches sur la dynamique de l'occupation et de l'utilisation des terres ont pu conclure à une régression des formations végétales au profit des formations anthropiques et naturelles (Shiferaw, 2011), notamment les espaces agricoles, il importe toutefois de retenir que très peu de travaux ont orienté les analyses vers les scénarios prospectifs de la dynamique de l'occupation des terres dans les espaces de développement agricole du Bénin, malgré les outils modernes de modélisation. Dans ces espaces agricoles surtout caractérisés par une agriculture intensive, les changements locaux sont brusques et importants, tandis que les structures paysagères environnantes évoluent lentement à l'échelle régionale (Mama, 2014 ; Bamba, 2010 ; Bogaert et al., 2003). Pour territorialiser son développement agricole, le Bénin a créé 7 pôles (Présidence de la République du Bénin, PAG : 2016-2021). Le 7ème abrite un sous - pôle, "le territoire de la Lama" qui subit actuellement une pression anthropique liée à la culture de l'ananas et des plantations de palmier à huile. L'objectif de la présente étude est de modéliser la dynamique spatio-temporelle de l'occupation du sol et d'analyser les changements intervenus dans le territoire de la Lama, d'ici à l'horizon Bénin - Alafia 2025 et à l'agenda 2030 conformément aux cibles 2 et 15 des ODD (FAO- ODD, 2017). Pour atteindre cet objectif, il faut vérifier le scénario suivant: la dynamique spatiotemporelle induit des changements rapides, lents ou stables avec des taux de conversion variables qui déterminent les niveaux de fragmentation du paysage.

\section{Secteur d'étude}

Le secteur d'étude est le territoire de la Lama, situé au sud du Bénin dans le département de l'Atlantique entre $6^{\circ} 32^{\prime} 08^{\prime \prime}$ ' et $6^{\circ} 58^{\prime} 14^{\prime \prime}$ de latitude nord, et entre $1^{\circ} 59^{\prime} 04^{\prime \prime}$ et $2^{\circ} 28^{\prime} 06^{\prime \prime}$ de longitude est. Il constitue un espace 
intercommunal rassemblant trois communes (Allada, Toffo et Zè). Le territoire de la Lama est limité au nord par la commune de Zogbodomey, au nord - est par le lac Hlan et le fleuve Ouémé, à l'ouest par les communes de Lalo et de Bopa, au sud - ouest par le fleuve Couffo, à l'est par les communes de Bonou et d'Adjohoun, au sud - est par la rivière Sô, affluent du fleuve Ouémé, au sud par les communes d'Abomey - Calavi, Tori-Bossito et Kpomassè. Sa superficie de 179505,38 ha abrite une population totale de 336010 habitants (INSAE, 2016). L'agriculture y est la principale activité. Les formations géologiques sont constituées essentiellement de dépôts sabloargileux altérés en faciès de terre de barre. La pédologie est formée de sols faiblement ferrallitiques communément appelés terre de barre. Les sols hydromorphes à horizon superficiel gris et assez riche constituent le substrat des plans d'eau et des bas-fonds (Mairie de Porto-Novo, 2006). Le climat permet de distinguer deux (02) saisons de pluie (une grande saison de mars à juin et une petite saison de septembre à novembre) et deux (2) saisons sèches (de juillet à septembre puis de novembre à mars). La pluviosité annuelle moyenne est de 800 à $1000 \mathrm{~mm}$ (Djohy et al., 2017). Le couvert végétal est composé de quatre ensembles à savoir : la forêt claire et savane boisée, les savanes arborées et arbustives, les formations aquatiques et les plantations d'Elaeis guineensis (palmier à huile). Les forêts classées de la Lama (16250 ha) et de Djigbé (3441 ha) marquent le paysage naturel au nord du secteur d'étude (FAO-Bénin, 2010). À Toffo, les savanes arborée et arbustive sont parsemées de reliques de forêt et de plantation. La faune quant à elle est très variée, mais s'amenuise de jour en jour (figure 1).

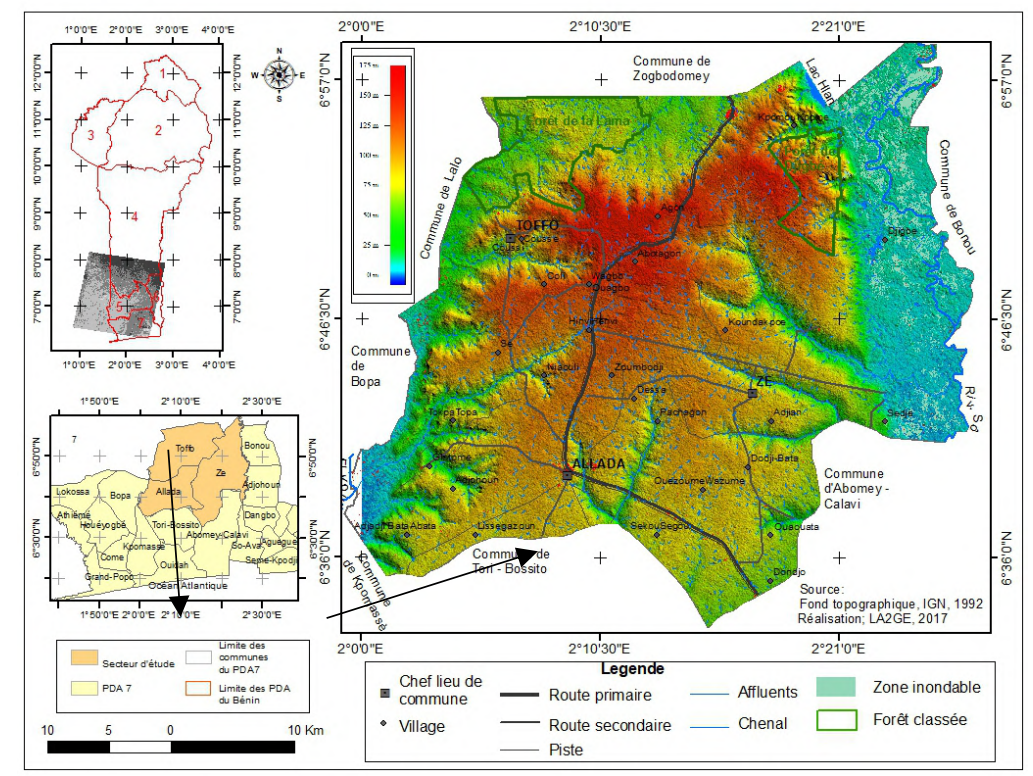

Figure 1 : Localisation du secteur d'étude

Source : Images SRTM 2015 et Landsat OLI, 2015 traitées, Tchibozo, 2019 
La lecture de la figure 1 montre que le relief du secteur d'étude est un plateau d'érosion d'une altitude moyenne de $100 \mathrm{~m}$ qui s'incline légèrement vers la côte et surplombe au Nord la dépression de la Lama. Il est disséqué par plusieurs vallées orientées $\mathrm{SW}-\mathrm{NE}$ et NW -SE et drainées par des chenaux fluviatiles reliant plusieurs affluents temporaires. Dans la commune de Toffo, à l'ouest du secteur d'étude, plusieurs talus à forte pente localisée témoignent le travail de l'érosion sur le rebord du plateau. Au pied de ce plateau, une plaine d'inondation recueille les eaux de ruissellement.

\section{Données et méthodes}

\section{Données utilisées}

Les données utilisées dans ce travail sont variées: littérature, recensements, cartes, images satellite, etc. Le tableau I en présente les principales caractéristiques spatiales qui ont déterminé la précision des résultats obtenus. 
Tableau I : principales caractéristiques des données images et des cartes thématiques

\begin{tabular}{|c|c|c|c|c|c|c|c|}
\hline $\mathbf{N}^{\circ}$ & Données & Sources et Format & $\begin{array}{l}\text { Échelle/ } \\
\text { Résolution } \\
\text { spatiale }\end{array}$ & $\begin{array}{l}\text { Résolution } \\
\text { spectrale des bandes } \\
\text { utilisées }\end{array}$ & Date & $\begin{array}{l}\text { Coordon } \\
\text {-nées de } \\
\text { la scène } \\
\end{array}$ & Utilisation \\
\hline 1 & $\begin{array}{l}\text { Image Landsat } 7, \\
\text { Radiomètre ETM+ }\end{array}$ & $\begin{array}{l}\text { USGS, } \\
\text { Earthexplorer } \\
\text { Raster }\end{array}$ & $30 \mathrm{~m}$ & $\begin{array}{l}\text { Multispectrale } \\
\text { B3-0.631- } 0.692 \mu \mathrm{m} \\
\text { B4-0.772- } 0.898 \mu \mathrm{m} \\
\text { B5-1.547- } 1.749 \mu \mathrm{m}\end{array}$ & Mars 2000 & $\begin{array}{l}192 / 055 \\
(\mathrm{WRS})\end{array}$ & Cartographie de l'occupation du sol \\
\hline \multirow[t]{3}{*}{2} & \multirow[t]{3}{*}{$\begin{array}{l}\text { Image Landsat } 8, \\
\text { Radiomètre } \\
\text { LDCM/OLI }\end{array}$} & & $30 \mathrm{~m}$ & $\begin{array}{l}\text { Multispectrale } \\
\text { B4- 0.636-0.673 } \mu \mathrm{m} \\
\text { B5- } 0.851-0.879 \mu \mathrm{m}\end{array}$ & \multirow[t]{3}{*}{$\begin{array}{l}\text { Janvier } \\
2015\end{array}$} & & \\
\hline & & & $60 \mathrm{~m}$ & B6- 1,566-1,651 $\mu \mathrm{m}$ & & & \\
\hline & & & $15 \mathrm{~m}$ & $\begin{array}{l}\text { Panchromatique } \\
\text { B8- 0.503-0.676 } \mu \mathrm{m}\end{array}$ & & & \\
\hline 3 & $\begin{array}{l}\text { Image SRTM, Shuttle } \\
\text { Radar Topography } \\
\text { Mission }\end{array}$ & $\begin{array}{l}\text { GLCF, } \\
\text { Raster }\end{array}$ & $30 \mathrm{~m}$ & - & $\begin{array}{l}\text { Janvier } \\
2015\end{array}$ & - & $\begin{array}{l}\text { Analyse du relief, cartographie des } \\
\text { pentes et des risques d'érosion }\end{array}$ \\
\hline 4 & $\begin{array}{l}\text { Carte topographique, } \\
\text { Feuille nb_31_20_21 } \\
\text { Zagnanado }\end{array}$ & $\begin{array}{l}\text { IGN, Bénin, } \\
\text { IGN, France, } \\
\text { Raster }\end{array}$ & $1 / 200000$ & - & 1955 & - & $\begin{array}{l}\text { Toponymie, hydronymie et travaux } \\
\text { de terrain }\end{array}$ \\
\hline 5 & $\begin{array}{l}\text { Carte touristique } \mathrm{du} \\
\text { Bénin }\end{array}$ & $\begin{array}{l}\text { IGN, Bénin, } \\
\text { IGN, France, } \\
\text { Raster }\end{array}$ & $1 / 600000$ & - & 1992 & - & Villages et voirie \\
\hline 5 & $\begin{array}{lll}\begin{array}{l}\text { Open } \\
(\text { OSM })\end{array} & \text { Street } & \text { Map } \\
\end{array}$ & OSM, Vecteur & - & - & 2017 & - & Mise à jour des données \\
\hline
\end{tabular}

Source : Recherche documentaire, septembre 2018 
Le matériel est constitué de plusieurs logiciels dont ENVI 5.3 pour la classification des images, ArcGIS 10.5 pour la vectorisation et l'édition cartographique, IDRISI Selva pour la modélisation prédictive, Global mapper 16 pour la modélisation des cours d'eau et IBM SPSS Statistic 21 pour les traitements statistiques. Un GPS est utilisé pour la prise de coordonnées des points sur le terrain.

\section{Méthode}

La méthodologie adoptée est basée sur une approche géographique et diachronique de l'étude de l'occupation du sol à partir du traitement des données de télédétection, de la cartographie par classification supervisée d'image, de la modélisation prédictive et de l'analyse spatiale. Les images du secteur d'étude sont extraites des scènes Landsat 7 ETM+ (2000) et Landsat 8 OLI/TIRS (2015). La composition colorée est réalisée par des combinaisons vraies couleur des bandes 5-4-3 pour l'image de Landsat 7 et 6-5-4 pour Landsat 8. Les images obtenues ont subi un traitement par l'égalisation de 1 'histogramme et filtrage avec $2 \%$ de saturation afin d'améliorer le contraste et faciliter ainsi l'interprétation de certaines zones de cultures et jachères proches des sols nus. À cette étape du traitement, les différentes images obtenues ont été interprétées avec la méthode visuelle et celle de la classification supervisée d'images (Singh, 1989) pour cartographier l'occupation du sol en 2000 et 2015. L'algorithme utilisé est celui du maximum de vraisemblance. Il calcule une fonction de probabilité multidimensionnelle pour déterminer la probabilité de chaque pixel d'appartenir à l'une des catégories correspondantes aux signatures spectrales (Benkouider, 2012; Abdel - Kawy et al., 2011). L'évaluation des résultats de la classification des images est réalisée à l'aide de la matrice de contingence ou matrice de confusion (Mugisha et al., 2010; Jensen, 1996). Des coordonnées GPS et des observations de terrain ont permis de valider la classification des images. La cartographie de la dynamique de l'occupation du sol est réalisée à partir de la vectorisation des résultats de la classification des images (raster). La dynamique spatiale est mesurée à partir de l'appréciation de l'évolution des états de surface fondée sur la comparaison des valeurs de superficie des différentes unités d'occupation du sol entre les périodes d'étude (Oloukoï et al., 2006). Les taux de conversion sont appréciés à partir de la mesure du degré de conversion d'une unité donnée en d'autres unités d'occupation du sol. Il est obtenu à partir de la matrice de transition (Arouna, 2012) suivant la formule ci-dessous :

$\mathrm{Tc}=[($ Sit - Sis $) /$ Sit $] \times 100$,

où Sit - Superficie de l'unité d'occupation du sol i à la date initiale $t$;

Sis - Superficie de la même unité i demeurée stable à la date $\mathrm{t}_{1}$. 
Les intensités de changement sont mesurées à l'aide du programme "PontiusMatrix22', (Pontius, 2000) pour obtenir une variété d'analyses et des statistiques sommaires sur la matrice de transition. La modélisation prospective de l'occupation du sol aux horizons 2025 et 2030 est faite à l'aide du logiciel "IDRISI Selva" suivant un scénario basé sur le modèle Markov. Cette modélisation s'est déroulée en trois phases : a) la constitution de la base de connaissances sur la dynamique spatio-temporelle de l'occupation du sol à partir de l'Évaluation MultiCritère (EMC) des variables environnementales ; b) le calcul des probabilités de transition par Analyse des Chaînes de Markov (ACM) entre 2000 et 2015 avec une date simulée (2030) qui a permis de mettre en évidence la moyenne des probabilités de transition obtenue pendant la période d'étude; c) l'allocation spatiale des probabilités de transition markoviennes : cette dernière étape utilise les résultats catégoriels de l'EMC. Le résultat de cette dernière phase est une carte d'occupation du sol des années simulées (2025 et 2030) du secteur d'étude. Le niveau de fragmentation du paysage est apprécié par le calcul de l'indice de fragmentation $\left(\mathrm{F}_{\mathrm{j}}\right)$ de chaque unité d'occupation du sol. Cette méthode appliquée de l'analyse spatiale mesure l'agrégation des pixels dans les classes t pour exprimer la complexité de l'image (Sambiéni et al., 2015 ; Bogaert et Hong, 2003). Il est calculé par la formule $F_{j}=\left(n_{j}-1\right) /\left(m_{j}-1\right)$,

où nj représente le nombre total des taches pour la classe $\mathrm{j} ; \mathrm{m}_{\mathrm{j}}$ est généralement dans un fichier raster, le nombre de pixels (Monmonier, 1974). Mais, le calcul ayant été effectué dans cette étude avec un fichier vectoriel, $\mathrm{m}_{\mathrm{j}}$ a été estimé par le rapport à l'aire totale $a_{j}$ de la classe $\mathrm{j}$ et l'aire de la plus petite tache $\left(a_{j} / a_{m i n j}\right)$. Donc $F_{j}=\left(n_{j}-1\right) /\left[\left(a_{t j} / a_{m i n j}\right)-1\right] ; 0 \leq F_{j} \leq 1$. Ainsi, la classe est moins fragmentée si $\mathrm{F}_{\mathrm{j}}$ est proche de 0 , et davantage fragmentée si $\mathrm{F}_{\mathrm{j}}$ est proche de 1 .

\section{Résultats}

$\mathrm{Au}$ terme de cette étude, cinq résultats sont obtenus : l'analyse de la dynamique spatio - temporelle de l'occupation du sol, l'analyse des changements, l'évaluation du taux de conversion des unités d'occupation du sol, la cartographie des intensités des changements et la mesure de la fragmentation du paysage.

\section{Analyse de la dynamique spatio - temporelle de l'occupation du sol}

La dynamique est étudiée à partir des cartes d'occupation du sol des années 2000 et 2015 et leur modélisation sur les périodes de 2025 et 2030 (figure 3). 


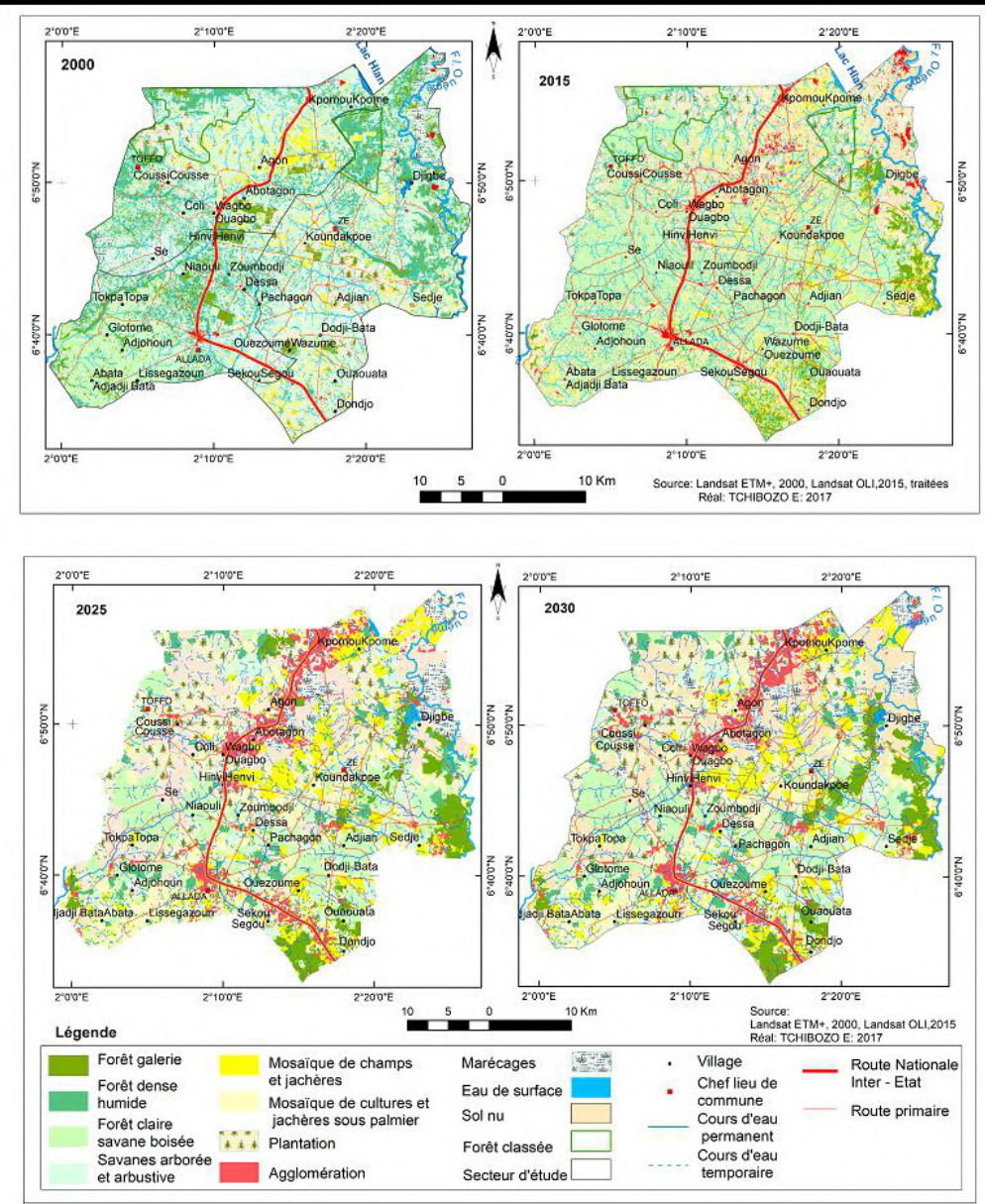

Figure 3 : Occupation du sol du territoire de la Lama en 2000, 2015, 2025 et 2030

L'histogramme comparatif des taux d'occupation du sol pour chacune des unités est présenté sur la figure 4 


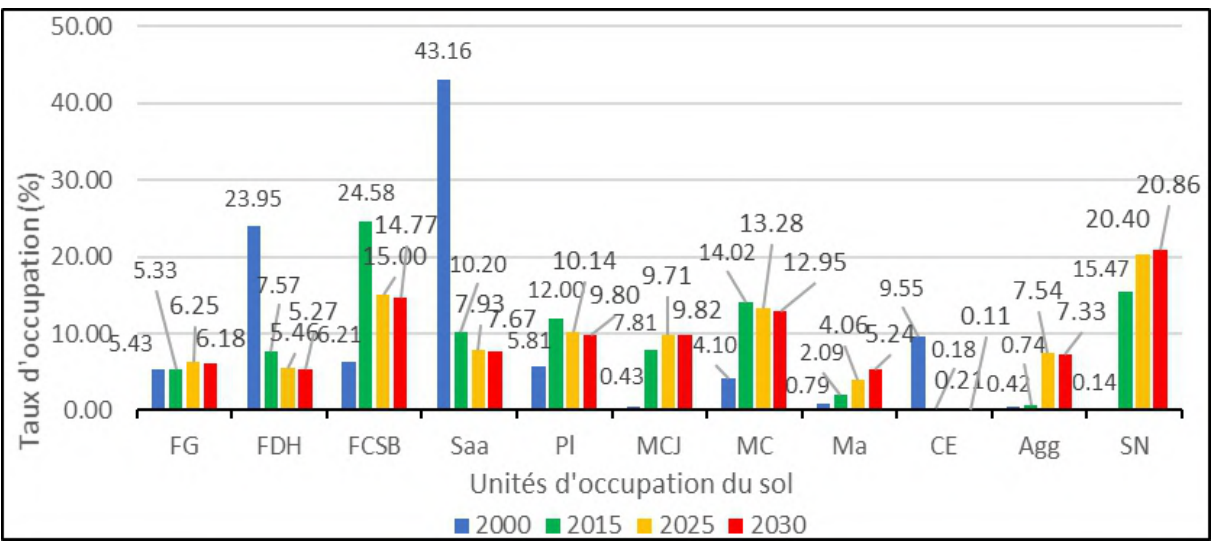

Figure 4 : Histogramme comparatif des taux d'occupation des unités

Source : Traitement statistique des résultats de cartographie, Tchibozo, 2017

Légende : FG - Forêt galerie ; FDH -Forêt dense humide ; FCSB- Forêt claire savane boisée ; Saa -Savanes arborée et arbustive ; Pl -Plantation ; MC- Mosaïque des cultures et jachères sous palmiers ; MCJ -Mosaïque de champs et jachères ; Ma -Marécages ; CE- Cours d'eau ; SN -Sol nu ; Agg - Agglomération.

L'analyse des figures 3 et 4 révèle une dynamique des unités d'occupation du sol pendant les périodes d'étude. On observe qu'en 2000, le paysage était codominé par les savanes arborée et arbustive $(43,16 \%)$ et la forêt dense humide $(23,95 \%)$. En 2015, cette hiérarchisation du paysage est réalisée cette fois -ci par la forêt claire et savane boisée à $24,58 \%$ et les sols nus à $15,47 \%$. En 2025, on verra la même situation précédemment illustrée en 2015 , mais avec une régression de $-38,97 \%$ pour la première unité d'occupation du sol et une progression de $+24,17 \%$ pour la deuxième. La forêt claire et savane boisée connaîtra une stabilité relative avec une faible régression de $-1,53 \%$. En 2030, on constatera la dominance du paysage essentiellement par les sols nus à $20,85 \%$. Cette situation révèle un recul des formations végétales et donc une dégradation progressive du paysage. Plus spécifiquement, on constate que la forêt-galerie a réalisé une faible régression de $-1,84 \%$ pendant la période de 2000 à 2015 , une progression de $+14,72 \%$ de 2015 à 2025 et une faible régression de $-1,12 \%$ de 2025 à 2030. La forêt dense humide a connu une régression consécutive de $-68,39 \%$ pour la première période, de $-27,87 \%$ pendant la deuxième et de $-3,48 \%$ durant la troisième. Les savanes arborée et arbustive ont suivi la même tendance que la forêt dense humide avec une régression significative de $-76,37 \%$ pour la période de 2000 à 2015, moindre de $-22,25 \%$ pour celle de 2015 à 2025 et une régression de $-3,28 \%$ de 2025 à 2030 . La forêt claire et savane boisée a montré une progression remarquable de $+74,74 \%$ pour la première période, une régression de $-38,97 \%$ pendant la deuxième et de $-1,53 \%$ au cours de la troisième période. Les marécages sont caractérisés par des progressions successives de leurs taux d'occupation de $+62,20 \%$ de 2000 à 2015 , de + 
$48,52 \%$ de 2015 à 2025 et de $+22,53 \%$ de 2025 à 2030 . Les agglomérations ont suivi la même tendance que les marécages avec une progression de $+43,24$ $\%$ pendant la première période, de $+90,19 \%$ durant la deuxième et une faible régression de $-2,79 \%$ au cours de la troisième période. Les sols nus montrent une progression récurrente successivement de $90,50 \%$, de $24,17 \%$ et de 2,20 $\%$ pour les périodes d'étude. La dynamique des eaux de surface est caractérisée par une régression sensible de $-82,15 \%$ de 2000 à 2015 , une progression de $14,28 \%$ de 2015 à 2025 et une régression de $-47,42 \%$ de 2025 à 2030. Les activités agricoles sont représentées par les plantations $(5,81 \%)$, la mosaïque de champs et jachères $(0,43 \%)$ et la mosaïque de cultures et jachères sous palmier $(4,10 \%)$. Les premières ont connu une progression de + $51,58 \%$ de 2000 à 2015 , une régression de $-15,50 \%$ de 2015 à 2025 et une faible progression de $1,12 \%$ de 2025 à 2030. Les deuxièmes montrent une progression significative de $+94,49 \%$ de 2000 à 2015 , de $+70,76 \%$ de 2015 à 2025 et moindre de $+19,57 \%$ de 2025 à 2030 . Les troisièmes ont effectué une progression distinctive de $+70,76 \%$ de 2000 à 2015 et une régression de $-5,28 \%$ de 2015 à 2025. De 2025 à 2030, cette unité d'occupation du sol exprimera une régression de $-2,48 \%$.

Ainsi de 2000 à 2015, la dynamique de l'occupation du sol est caractérisée par une faible régression de la forêt-galerie et importante de la forêt dense humide et des savanes arborée et arbustive. On note une progression importante de la forêt claire et savane boisée pendant cette période. Les marécages, les agglomérations et les sols nus ont progressé alors que les eaux de surface ont régressé sensiblement. On constate que les plantations ont connu une progression importante de même que la mosaïque de champs et jachères et celle de cultures et jachères sous palmier.

De 2015 à 2025, la forêt-galerie et la forêt claire et savane boisée vont progresser alors que la forêt dense humide et les savanes arborée et arbustive connaîtront une régression. Les marécages, les agglomérations et les sols nus connaitront une augmentation sensible de leur taux d'occupation alors que les eaux de surface vont suivre la même tendance que les unités précédentes, mais avec une augmentation modérée. Les plantations montreront une régression de même que la mosaïque de cultures et jachères sous palmier alors que celle de champs et jachères connaîtra une progression sensible.

De 2025 à 2030, on observera que toutes les formations végétales exprimeront une faible régression. Les marécages deviendront plus importants alors que les agglomérations et les sols nus vont diminuer faiblement. Les eaux de surface connaîtront une réduction remarquable alors que les plantations et la mosaïque de champs et jachères manifesteront une progression. La mosaïque de cultures et jachères sous palmier sera caractériser par une faible régression. 


\section{Identification des changements dans l'occupation du sol}

Pour l'étude diachronique, les changements observés sur chaque unité d'occupation du sol caractérisent leurs dynamiques dans le paysage. Les tableaux 2, 3 et 4 en exposent les valeurs pour les périodes de 2000 à 2015, 2015 à 2025 et 2025 à 2030.

Tableau II : Matrice de transition des unités d'occupation du sol entre 2000 et 2015

\begin{tabular}{|l|l|l|l|l|l|l|l|l|l|l|l|l|}
\hline & FG & FDH & FCSB & SaA & PI & MCJ & MC & Ma & CE & Agg & SN & $\begin{array}{l}\text { Total } \\
\mathbf{2 0 0 0} \text { (ha) }\end{array}$ \\
\hline FG & $\mathbf{1 1 , 2 5}$ & 0,00 & 0,00 & 0,00 & 41,13 & 104,40 & 31,05 & 1,17 & 5,85 & 0 & 11,25 & $\mathbf{2 0 6 , 1}$ \\
\hline FDH & 0,00 & $\mathbf{8 2 , 8 9}$ & 257,04 & 92,07 & 137,25 & 45,09 & 115,83 & 10,53 & 0 & 1,44 & 112,59 & $\mathbf{9 0 9 , 2 7}$ \\
\hline FCSB & 0,00 & 13,68 & $\mathbf{2 5 , 0 2}$ & 18,54 & 31,86 & 23,4 & 38,34 & 7,29 & 0 & 1,89 & 62,28 & $\mathbf{2 3 5 , 8 9}$ \\
\hline Saa & 0,00 & 123,75 & 335,34 & $\mathbf{1 9 8 , 9}$ & 188,28 & 132,21 & 264,96 & 34,38 & 0,09 & 7,38 & 279,81 & $\mathbf{1 6 3 8 , 3 6}$ \\
\hline PI & 75,96 & 7,92 & 25,56 & 18,54 & $\mathbf{1 9 , 9 8}$ & 29,07 & 32,94 & 10,08 & 0 & 5,22 & 49,68 & $\mathbf{2 2 0 , 4 1}$ \\
\hline MCJ & 15,57 & 0,63 & 2,52 & 0,81 & 1,71 & $\mathbf{2 , 6 1}$ & 1,35 & 0,9 & 0 & 0,72 & 2,97 & $\mathbf{1 6 , 2}$ \\
\hline MC & 83,88 & 9,72 & 25,02 & 15,12 & 13,32 & 17,1 & $\mathbf{2 2 , 8 6}$ & 5,58 & 0 & 1,8 & 34,47 & $\mathbf{1 5 5 , 6 1}$ \\
\hline Ma & 0,63 & 1,35 & 3,06 & 2,79 & 2,61 & 3,24 & 4,95 & $\mathbf{1 , 3 5}$ & 0,09 & 1,35 & 8,73 & $\mathbf{3 0 , 1 5}$ \\
\hline CE & 14,76 & 29,79 & 169,11 & 28,53 & 36,81 & 21,87 & 30,87 & 1,89 & $\mathbf{0 , 9}$ & 0,36 & 27,45 & $\mathbf{3 6 2 , 3 4}$ \\
\hline Agg & 0,09 & 0 & 0,09 & 0 & 0,18 & 0,27 & 0,45 & 5,31 & 0,09 & $\mathbf{5 , 4 9}$ & 4,05 & $\mathbf{1 6 , 0 2}$ \\
\hline SN & 0,18 & 0 & 0 & 0 & 0,09 & 0,45 & 0,36 & 0,9 & 0 & 2,25 & $\mathbf{1 , 2 6}$ & $\mathbf{5 , 4 9}$ \\
\hline $\begin{array}{l}\text { Total } \\
\mathbf{2 0 1 5} \text { (ha) }\end{array}$ & $\mathbf{2 0 2 , 3 2}$ & $\mathbf{2 8 7 , 3 7}$ & $\mathbf{9 3 2 , 9 4}$ & $\mathbf{3 8 7 , 1 8}$ & $\mathbf{4 5 5 , 5 8}$ & $\mathbf{2 8 9 , 5 3}$ & $\mathbf{5 3 2 , 0 8}$ & $\mathbf{7 9 , 3 8}$ & $\mathbf{7 , 0 2}$ & $\mathbf{2 7 , 9}$ & $\mathbf{5 9 4 , 5 4}$ & $\mathbf{3 7 9 5 , 8 4}$ \\
\hline
\end{tabular}

Source : Traitement statistique des résultats de cartographie, Tchibozo, 2019

Tableau III : Matrice de transition des unités d'occupation du sol entre 2015 et 2025

\begin{tabular}{|l|l|l|l|l|l|l|l|l|l|l|l|l|}
\hline & FG & FDH & FCSB & Saa & PI & MCJ & MC & Ma & CE & Agg & SN & $\begin{array}{l}\text { Total } \\
\text { (ha) }\end{array}$ \\
\hline FG & $\mathbf{1 0 8 , 6 3}$ & 0,00 & 0,00 & 0,00 & 8,28 & 152,55 & 2,13 & 0,00 & 7,02 & 0,00 & 0,00 & $\mathbf{2 0 2 , 3 2}$ \\
\hline FDH & 0,00 & $\mathbf{1 2 1 , 8 6}$ & 63,45 & 19,98 & 45 & 0,36 & 23,67 & 0,00 & 0,00 & 0,00 & 9,09 & $\mathbf{2 8 7 , 4 6}$ \\
\hline FCSB & 0,00 & 31,23 & $\mathbf{3 1 3 , 1 1}$ & 33,66 & 110,25 & 50,4 & 131,4 & 1,35 & 0,00 & 0,00 & 252,18 & $\mathbf{9 3 3 , 1 2}$ \\
\hline Saa & 0,00 & 21,87 & 48,15 & $\mathbf{1 9 2 , 6}$ & 11,97 & 14,4 & 58,14 & 0,45 & 0,00 & 0,00 & 34,11 & $\mathbf{3 8 7 , 3 6}$ \\
\hline PI & 32,85 & 1,71 & 13,5 & 10,71 & $\mathbf{1 9 6 , 9 2}$ & 32,13 & 48,51 & 11,52 & 0,00 & 0,00 & 111,78 & $\mathbf{4 5 5 , 5 8}$ \\
\hline MCJ & 50,58 & 1,98 & 11,97 & 0,63 & 4,5 & $\mathbf{2 0 3 , 0 4}$ & 1,62 & 10,17 & 0,00 & 0,54 & 20,79 & $\mathbf{2 9 6 , 2 8}$ \\
\hline MC & 70,65 & 19,44 & 38,52 & 38,88 & 15,57 & 52,02 & $\mathbf{2 3 1 , 2 1}$ & 5,94 & 0,00 & 0,00 & 98,01 & $\mathbf{5 3 2 , 0 8}$ \\
\hline Ma & 0,00 & 0,54 & 0,27 & 2,7 & 0,63 & 2,7 & 6,93 & $\mathbf{4 1 , 1 3}$ & 0,00 & 0,00 & 24,48 & $\mathbf{7 9 , 3 8}$ \\
\hline CE & 0,00 & 0,27 & 4,86 & 0,81 & 0,18 & 0,00 & 0,00 & 0,00 & $\mathbf{0 , 9 0}$ & 0,00 & 0,00 & $\mathbf{7 , 0 2}$ \\
\hline Agg & 0,00 & 0,00 & 0,00 & 0,00 & 0,00 & 0,00 & 0,00 & 10,26 & 0,00 & $\mathbf{1 7 , 3 7}$ & 0,27 & $\mathbf{2 7 , 9}$ \\
\hline SN & 0,00 & 0,54 & 0,00 & 0,36 & 0,00 & 19,26 & 1,80 & 73,26 & 0,00 & 268,29 & $\mathbf{2 2 3 , 8 3}$ & $\mathbf{5 8 7 , 3 4}$ \\
\hline $\begin{array}{l}\text { Total } \\
\mathbf{2 0 2 5} \\
\text { (ha) }\end{array}$ & $\mathbf{2 3 0 , 2 2}$ & $\mathbf{2 0 7 , 3 6}$ & $\mathbf{5 6 9 , 5 2}$ & $\mathbf{3 0 0 , 9 6}$ & $\mathbf{3 8 5 , 3 8}$ & $\mathbf{3 7 5 , 4 8}$ & $\mathbf{5 0 4 , 1 8}$ & $\mathbf{1 5 4 , 0 8}$ & $\mathbf{7 , 9 2}$ & $\mathbf{2 8 6 , 2}$ & $\mathbf{7 7 4 , 5 4}$ & $\mathbf{3 7 9 5 , 8 4}$ \\
\hline
\end{tabular}

Source : Traitement statistique des résultats de cartographie, Tchibozo, 2019 
Tableau IV : Matrice de transition des unités d'occupation du sol entre 2025 et 2030

\begin{tabular}{|l|l|l|l|l|l|l|l|l|l|l|l|l|}
\hline & & & & & & & & & & & & $\begin{array}{l}\text { Total } \\
\mathbf{2 0 2 5} \\
\text { (ha) }\end{array}$ \\
\hline FG & $\mathbf{1 7 6 , 4 9}$ & 0,00 & 0,00 & 0,00 & 14,40 & 18,45 & 11,34 & 0,18 & 0,00 & 0,90 & 15,66 & $\mathbf{2 3 7 , 4 2}$ \\
\hline FDH & 0,00 & $\mathbf{1 5 6 , 8 7}$ & 5,40 & 3,51 & 2,61 & 7,29 & 8,64 & 2,34 & 0,00 & 0,00 & 16,65 & $\mathbf{2 0 7 , 3 6}$ \\
\hline FCSB & 0,00 & 15,12 & $\mathbf{4 6 0 , 4}$ & 17,91 & 3,69 & 11,61 & 12,78 & 4,32 & 0,27 & 1,17 & 8,37 & $\mathbf{5 6 9 , 5 2}$ \\
\hline Saa & 0,00 & 3,6 & 7,65 & $\mathbf{2 4 6 , 0 6}$ & 4,23 & 7,02 & 11,25 & 2,97 & 0,09 & 1,44 & 11,88 & $\mathbf{3 0 0 , 9 6}$ \\
\hline PI & 9,81 & 11,07 & 24,39 & 1,62 & $\mathbf{3 0 9 , 4}$ & $\mathbf{4 , 2 3}$ & 4,86 & 2,97 & 0,09 & 6,66 & 13,86 & $\mathbf{3 8 4 , 9 3}$ \\
\hline MCJ & 36,63 & 0,72 & 8,64 & 5,31 & 6,48 & 304,83 & $\mathbf{1 4 , 4 9}$ & 4,86 & 0,00 & 10,08 & 11,61 & $\mathbf{3 6 8 , 7 3}$ \\
\hline MC & 7,47 & 5,58 & 22,23 & 10,62 & 11,7 & 11,16 & 416,3 & $\mathbf{5 , 5 8}$ & 0,00 & 3,78 & 14,58 & $\mathbf{5 0 4 , 1 8}$ \\
\hline Ma & 1,35 & 0,00 & 1,71 & 0,00 & 1,26 & 0,99 & 0,18 & 129,6 & $\mathbf{0 , 0 0}$ & 10,71 & 8,28 & $\mathbf{1 5 4 , 0 8}$ \\
\hline CE & 2,16 & 0,27 & 0,27 & 0,00 & 0,00 & 0,00 & 0,81 & 0,00 & 3,87 & $\mathbf{0 , 0 0}$ & 0,54 & $\mathbf{7 , 9 2}$ \\
\hline Agg & 0,00 & 0,00 & 0,00 & 0,00 & 0,00 & 0,00 & 0,00 & 14,76 & 0,00 & 218,1 & $\mathbf{5 3 , 3 7}$ & $\mathbf{2 8 6 , 2}$ \\
\hline SN & 1,8 & 6,03 & 27,00 & 3,78 & 18,99 & 10,08 & 13,23 & 31,5 & 0,00 & 25,29 & 636,8 & $\mathbf{7 7 4 , 5 4}$ \\
\hline $\begin{array}{l}\text { Total } \\
\text { 2030 } \\
\text { (ha) }\end{array}$ & & & & & & & & & & & & \\
\end{tabular}

Source : Traitement statistique des résultats de cartographie, Tchibozo, 2019

NB. Les cellules en gris représentent les unités conservées dans l'occupation du sol

Légende : FG - Forêt galerie ; FDH -Forêt dense humide ; FCSB- Forêt claire savane boisée ; Saa -Savanes arborée et arbustive ; Pl -Plantation ; MC- Mosaïque des cultures et jachères sous palmiers ; MCJ -Mosaïque de champs et jachères ; Ma -Marécages ; CE- Cours d'eau ; SN -Sol nu ; Agg - Agglomération.

L'analyse des tableaux II, II et IV révèle des changements au niveau de plusieurs unités d'occupation du sol dont les plus importants caractérisent des pertes et des gains surtout pour la forêt claire et savane boisée de même que les savanes arborée et arbustive. Ainsi, de 2000 à 2015, une superficie de 257,04 ha $(28,27 \%)$ de forêt dense humide s'est transformée en forêt claire et savane boisée, 137,25 ha $(15,09 \%)$ en plantation, 115,83 ha $(12,74 \%)$ en mosaïque de cultures et jachères sous palmier et 1212,59 ha $(12,38 \%)$ en sols nus (régression). De même, une étendue de 335,34 ha (20,47 \%) de savanes arborée et arbustive est changée en forêt claire et savane boisée, 279,81 ha $(17,08 \%)$ en sols nus, 264,96 ha $(16,17 \%)$ en mosaïque de cultures et jachères sous palmier, 188,28 ha $(11,49 \%)$ en plantations, 132,21 ha $(8,07 \%)$ en mosaïque de champs et jachères et 123,75 ha $(7,55 \%)$ en forêt dense humide (progression).

De 2015 à 2025, les changements les plus importants seront observés au niveau de la forêt claire et savane boisée ainsi qu'au niveau des sols nus. Subséquemment, 252,18 ha $(27,02 \%)$ de forêt claire et savane boisée seront converties en sols nus, 131,40 ha $(14,08 \%)$ en mosaïque de cultures et jachères sous palmier et 110,25 ha $(11,81 \%)$ en plantations. Il faut remarquer aussi que 268,29 ha (45,68\%) des sols nus seront transformés en agglomérations. 
De 2025 à 2030, la plupart des unités d'occupation montreront une conservation de leur taux d'occupation et donc n'exprimeront pas de changement important.

\section{Évaluation du taux de conversion des unités d'occupation du sol}

La figure 5 présente l'histogramme comparatif des taux de conversion des unités d'occupation du sol pendant les périodes d'étude

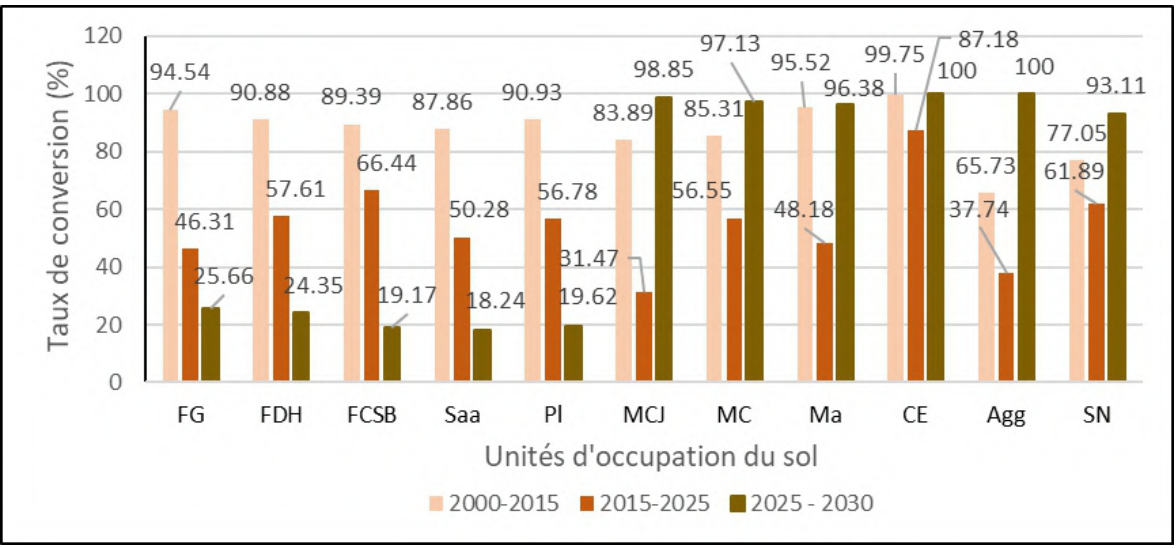

Figure 5 : Histogramme comparatif des taux de conversion des unités d'occupation du sol

Source : Traitement statistique des résultats de cartographie, Tchibozo, 2019

Légende : FG - Forêt galerie; FDH -Forêt dense humide ; FCSB- Forêt claire savane boisée ; Saa -Savanes arborée et arbustive ; Pl -Plantation ; MC- Mosaïque des cultures et jachères sous palmiers ; MCJ -Mosaïque de champs et jachères ; Ma -Marécages ; CE- Cours d'eau ; SN -Sol nu ; Agg - Agglomération.

L'analyse de cette figure montre que les taux de conversion varient suivant les périodes d'étude et les unités d'occupation du sol considérées. Les valeurs les plus importantes de ce taux sont observées pendant la période de 2025 à 2030.

De 2000 à 2015, on observe que les valeurs les plus élevées de cet indicateur caractérisent les eaux de surface $(99,75 \%)$, les marécages $(95,52$ $\%)$, la forêt-galerie $(94,54 \%)$ et la forêt dense humide $(90,88 \%)$. Cette situation révèle que les changements intervenus au niveau de ces unités d'occupation du sol sont rapides. Les taux de conversion les moins élevés pendant cette période sont remarqués sur les agglomérations $(65,73 \%)$ et les sols nus $(77,05 \%)$ et révèlent un changement lent sur ces unités d'occupation du sol.

De 2015 à 2025, les taux de conversion élevés caractérisent les eaux de surface $(87,18 \%)$, la forêt claire et savane boisée $(66,44 \%)$, et les sols nus $(61,89 \%)$. Cette disposition montre les changements rapides intervenus sur ces unités d'occupation du sol. Les valeurs peu significatives de ce taux sont remarquées sur les agglomérations $(37,74 \%)$ et la mosaïque des champs et 
jachères $(31,47 \%)$ et révèlent que les changements sur ces unités d'occupation sont lents.

De 2025 à 2030, les proportions dominantes du taux de conversion sont admises sur les eaux de surface (100\%), les agglomérations (100\%), la mosaïque des champs et jachères $(98,85 \%)$, celle des cultures et jachères sous palmier $(97,13 \%)$, les marécages $(96,38 \%)$ et les sols nus $(93,11 \%)$. Les plus faibles valeurs de ce taux caractérisent les formations végétales comme la forêt-galerie $(25,66 \%)$, la forêt dense humide $(24,35 \%)$, la forêt claire et savane boisée $(19,17 \%)$ et les savanes arborée et arbustive $(18,24 \%)$.

\section{Cartographie des intensités des changements des unités d'occupation du sol}

Sur le plan spatial, les changements dans l'occupation du sol se sont opérés de façon différentielle dans le secteur d'étude. La quantification des intensités de changement pendant les périodes d'étude est illustrée par les figures $6,7,8,9,10,11$ ci-dessous.

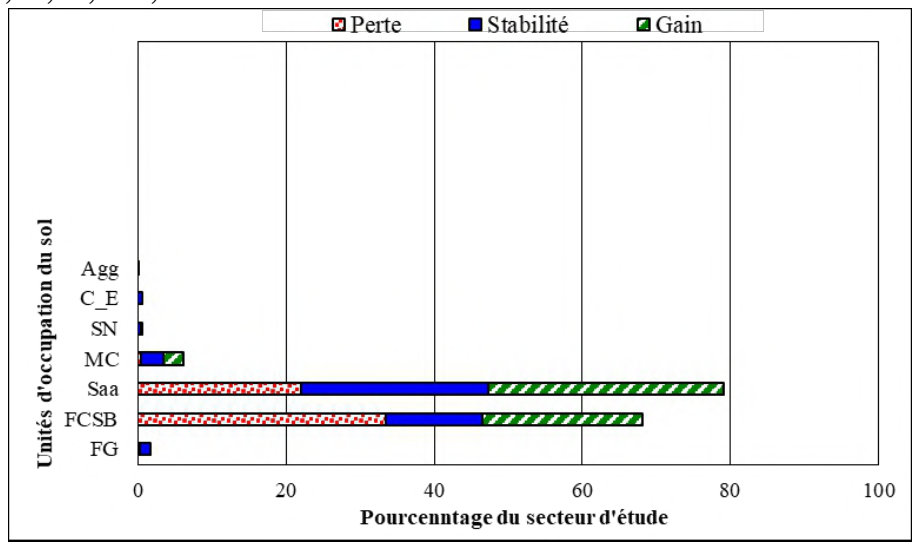

Figure 6 : Intensité des changements opérés par unité d'occupation du sol entre 2000 et 2015

Source : Traitement statistique de la matrice de transition, Tchibozo, 2019

Légende : Agg - Agglomération; C_E- Cours d'eau; SN-Sols nus ; MC- Mosaïque des cultures et jachères sous palmiers ; Saa - Savane arborée et arbustive ; FCSB- Forêt claire savane boisée ; FG- Forêt galerie.

L'analyse de la figure 6 révèle une perte plus importante pour la forêt claire et savane boisée $(33,49 \%$, soit 1271,22 ha) que pour les savanes arborée et arbustive (25,22\%, soit 957,31 ha). Les gains sont relativement importants pour les savanes arborée et arbustive $(31,86 \%$, soit 1209,35 ha) que pour la forêt claire et savane boisée $(21,66 \%$, soit 822,18 ha). La stabilité représente $25,32 \%$ (soit 861,11 ha) pour les savanes arborée et arbustive contre 13,01\% (soit 493,84 ha) pour la forêt claire et savane boisée pour la période de 2000 à 2015. La figure 7 présente l'intensité des changements en fonction des gains et des pertes enregistrés sur les unités d'occupation du sol de 2000 à 2015. 


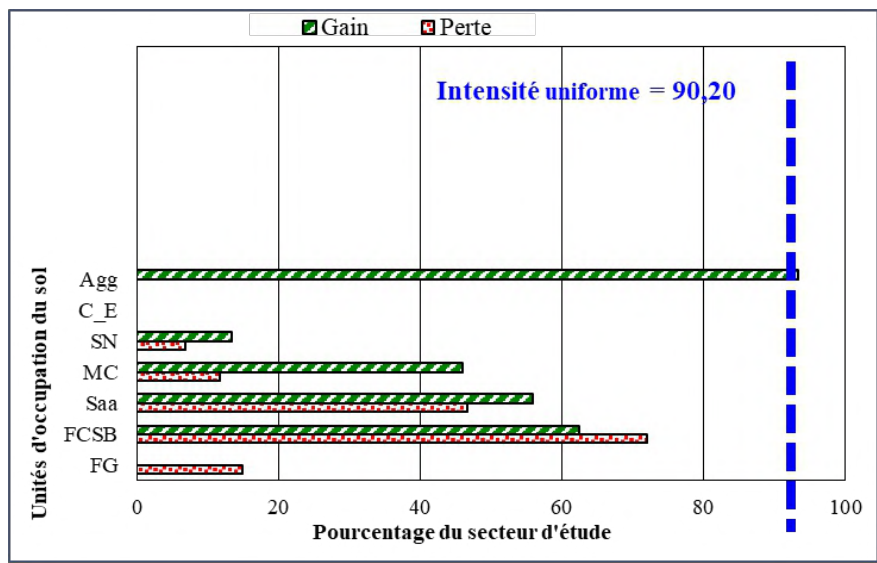

Figure 7 : Intensité des changements en fonction des gains et des pertes enregistrés sur les unités d'occupation du sol de 2000 à 2015

Source : Traitement statistique de la matrice de transition, Tchibozo, 2019

L'analyse de la figure 7 montre que sur la période de 2000 à 2015, toutes les unités d'occupation du sol ont connu des changements sous forme de pertes et de gains par rapport à leur pourcentage initial. Comme dans le cas précédent, les changements opérés sur les différentes unités d'occupation du sol sont tous actifs ou rapides. Dans l'ensemble, le milieu d'étude est caractérisé par une grande vitesse de changement sur les unités d'occupation du sol. L'intensité uniforme des changements se réalise à une vitesse de 90,20 $\%$ par an. De ce qui précède, on peut noter que les gains observés sur les agglomérations représentent 93,34\% du secteur d'étude soit 3568,09 ha, ceux de la forêt claire et savane boisée constituent $62,46 \%$ soit 2370,88 ha, les savanes arborée et arbustive symbolisent $55,81 \%$ soit 2118,46 ha, la mosaïque de cultures et jachères sous palmier occupe $45,98 \%$ soit 1745,33 ha et les sols nus $13,34 \%$ soit 506,37 ha. Les pertes sont constatées sur la forêt claire et savane boisée représentent $72,01 \%$ du secteur d'étude soit 2733,38 ha, les savanes arborée et arbustive 46,67 \% soit 1771,52 ha, la forêt-galerie 14,88 \% soit 564,82 ha, la mosaïque de cultures et jachères sous palmier $11,62 \%$ soit 441,08 ha et les sols nus $6,88 \%$ soit 261,15 ha. L'état de rapidité des changements est indiqué par la ligne verticale en tireté bleu, appelé ligne de zone uniforme. Les changements rapides caractérisent la forêt claire et savane boisée, les savanes arborée et arbustive et les agglomérations. Les autres unités d'occupation du sol expriment un changement lent ou dormant. La figure 8 présente l'intensité des changements opérés par unité d'occupation du sol entre 2015 et 2025. 


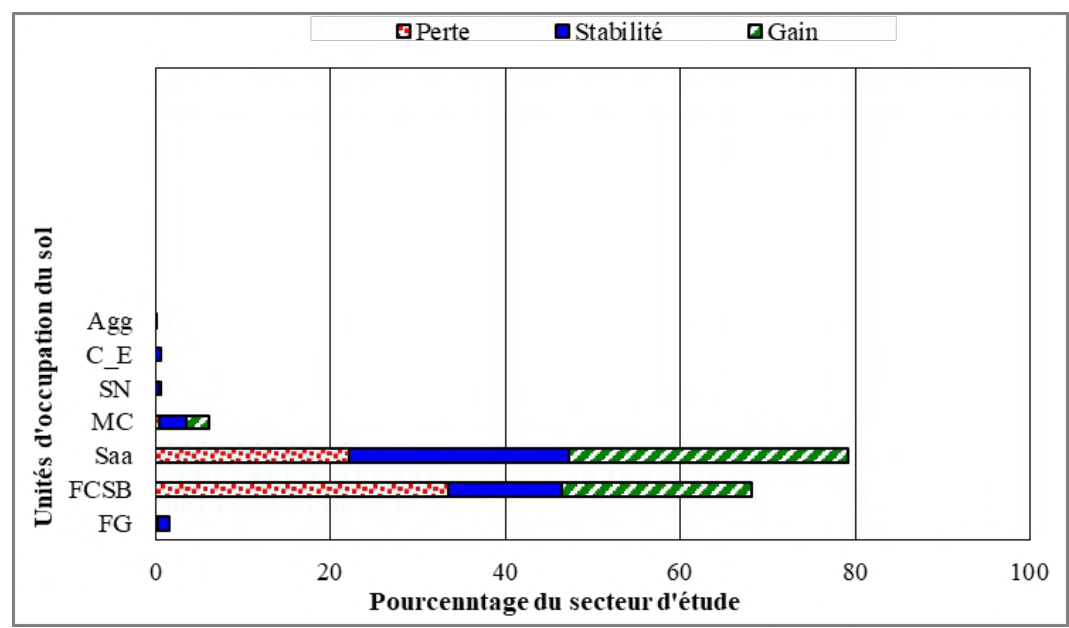

Figure 8 : Intensité des changements opérés par unité d'occupation du sol entre 2015 et 2025

Source : Traitement statistique de la matrice de transition, Tchibozo, 2019

Sur la figure 8, on constate que la période 2015 à 2025 est caractérisée par une intensité des changements rapides, plus importante pour les savanes arborée et arbustive que pour la forêt claire et savane boisée. Pour la première unité, les pertes représentent 22,07\% (soit 837,74 ha) alors qu'au niveau de la deuxième unité, elles constituent 33,49\% (soit 1271,23 ha). Les gains sont respectivement de $31,86 \%$ (soit 120 935,46 ha) et 21,66\% (soit 822,18 ha) pour ces deux formations végétales. La stabilité occupe $25,22 \%$ du secteur d'étude pour les savanes arborée et arbustive et 13,01\% pour la forêt claire et savane boisée.

La figure 9 illustre l'intensité des changements en fonction des gains et des pertes enregistrés sur les unités d'occupation du sol de 2015 à 2025.

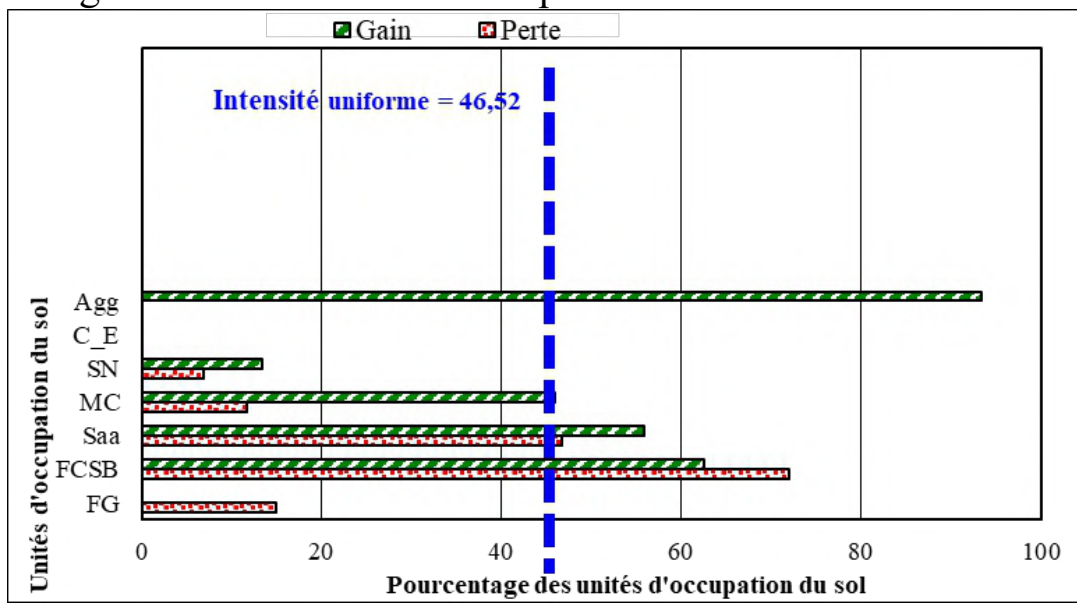

Figure 9 : Intensité des changements en fonction des gains et des pertes enregistrés sur les unités d'occupation du sol de 2015 à 2025

Source : Traitement statistique de la matrice de transition, Tchibozo, 2019 
L'analyse de la figure 9 révèle que sur la période de 2015 à 2025, les changements opérés sur les différentes unités d'occupation du sol sont moins rapides que ceux constatés sur la période de 2000 à 2015. Le milieu est caractérisé par une vitesse moyenne de changement des unités d'occupation du sol évaluée à 46,52 \% par an. Singulièrement, il faut noter que les agglomérations subissent un changement plus rapide que les autres unités d'occupation du sol avec un gain de $93,34 \%$, soit 3549,04 ha, de $62,46 \%$ soit 2370,88 ha pour la forêt claire et savane boisée, de $55,81 \%$, soit 218,46 ha pour les savanes arborée et arbustive, de 45,98 \% soit 1745,33 ha pour la mosaïque de champs et jachères et de $13,34 \%$ soit 506,37 ha pour les sols nus. Les pertes représentent $72,01 \%$ soit 2733,38 ha pour la forêt claire et savane boisée, $55,81 \%$ soit 2118,46 ha pour les savanes arborée et arbustive, 14,88 $\%$ soit 564,82 ha pour la forêt-galerie, $11,62 \%$ soit 441,08 ha pour la mosaïque de champs et jachères et $6,88 \%$ soit 261,15 ha pour les sols nus. La figure 10 présente l'intensité des changements opérés par unité d'occupation du sol entre 2025 et 2030.

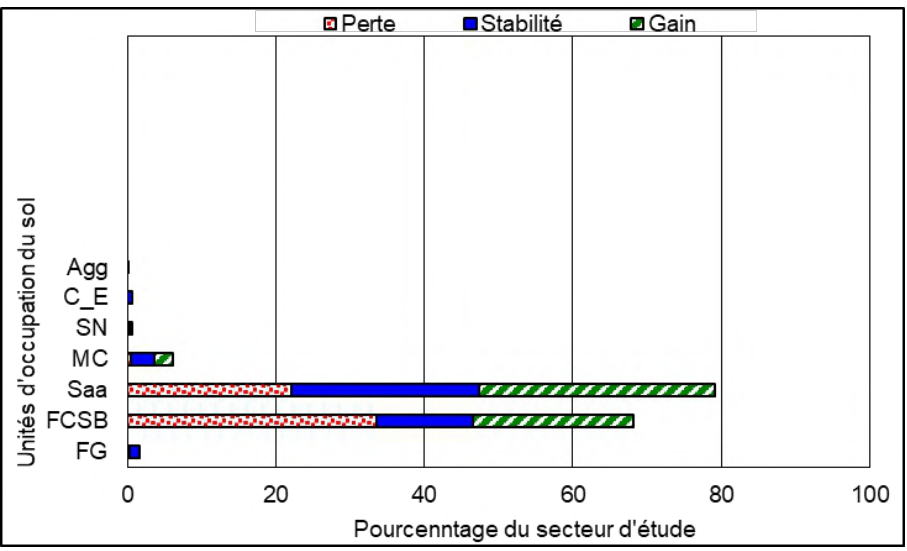

Figure 10 : Intensité des changements opérés par unité d'occupation du sol entre 2025 et 2030

Source : Traitement statistique de la matrice de transition, Tchibozo, 2019

Sur la figure 10, on constate que la période de 2025 à 2030 est caractérisée par une intensité des changements rapides et plus importants pour les savanes arborée et arbustive que pour la forêt claire et savane boisée. Les premières expriment un gain de $31,86 \%$ alors que pour le deuxième, cette valeur ne représente que $21,66 \%$. Les pertes constituent respectivement 22,07 $\%$ pour les savanes arborée et arbustive, $33,49 \%$ pour la forêt claire et savane boisée. La stabilité représente $25,33 \%$ pour les premières unités, $13,01 \%$ pour la deuxième et 3,09 \% pour la mosaïque de champs et jachères. La figure 11 illustre l'intensité des changements en fonction des gains et des pertes enregistrés sur les unités d'occupation du sol de 2025 à 2030. 


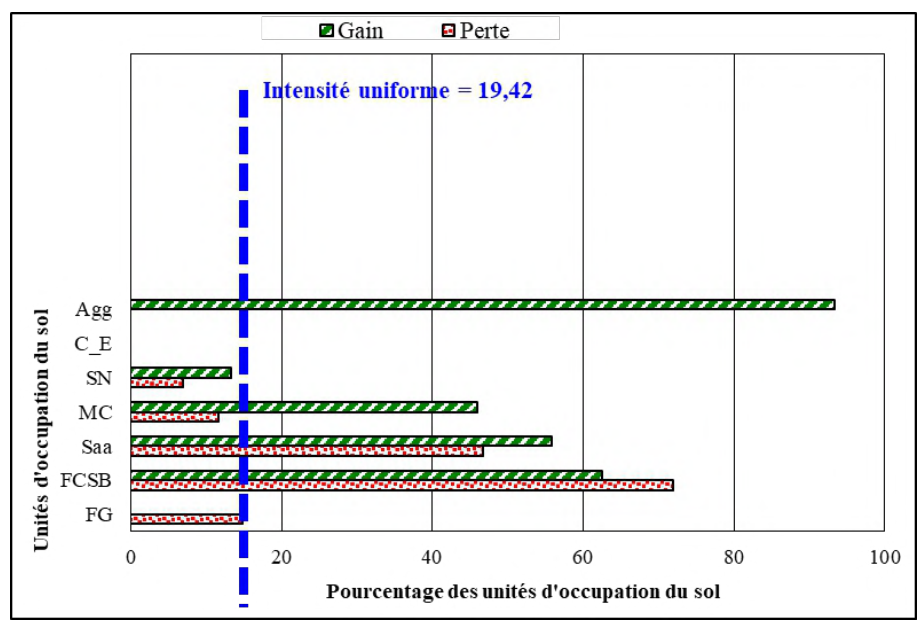

Figure 11 : Intensité des changements en fonction des gains et des pertes enregistrés sur les unités

d'occupation du sol de 2025 à 2030

Source : Traitement statistique de la matrice de transition, Tchibozo, 2019

Sur la figure 11, l'intensité uniforme des changements représente 19,42 \% du secteur d'étude. On constate que les changements rapides de l'agglomération ont généré un gain important de 93,34\% alors que la forêt claire et savane boisée en en exprime $62,46 \%$ et les savanes arborée et arbustive $55,81 \%$, la mosaïque de champs et jachères $45,98 \%$ et les sols nus $13,34 \%$. Les pertes constituent respectivement 72,01\% pour la forêt claire et savane boisée, 55,81\% pour les savanes arborée et arbustive, 11,62\% pour la mosaïque de champs et jachères et $6,88 \%$ pour les sols nus. Cette situation révèle un changement lent sur la forêt - galerie, la mosaïque de champs et jachères et les sols nus. La carte des intensités de changement observées dans le secteur d'étude pendant les périodes de 2000 à 2015 et 2015 à 2030 est présente la figure 12 . 


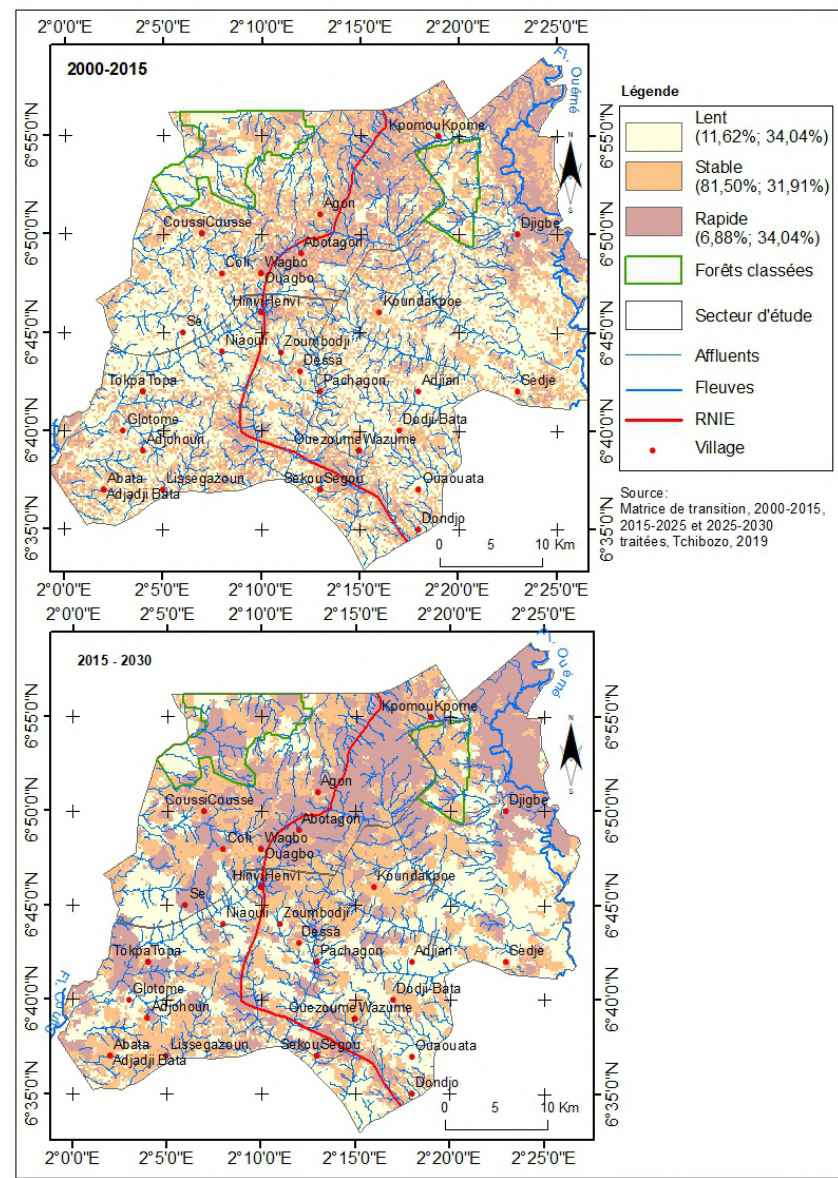

Figure 12 : Cartes des changements de 2000- 2015 et 2015-2030

Source : Traitements cartographique et statistique, 2019

L'analyse de la figure 12 montre que les vitesses de changement (lent, stable et rapide) sont inégalement réparties dans le secteur d'étude suivant la période considérée. De 2000 à 2015, les changements lents occupent 11,62\% du secteur d'étude, les changements rapides $6,88 \%$ et les changements stables $81,50 \%$. Les premiers couvrent généralement l'interfluve, les rebords ouest et est du plateau, le sud des vallées de l'Ouémé et du Couffo et les forêts classées de la Lama et de Djigbé. Les deuxièmes caractérisent aussi l'interfluve et les forêts classées alors que les troisièmes sont remarqués autour des agglomérations situées au nord de la Route Nationale Inter -États, au nord de la vallée de l'Ouémé et dans la forêt classée de la Lama.

De 2015 à 2030, les changements lents occuperont 34,04\% du secteur d'étude au même titre que les changements rapides. Les zones de changement stable représenteront $31,91 \%$.

De ce qui précède, on peut dire que dans le secteur d'étude, les changements lents ont augmenté de 65,86\% et ceux rapides de 79,79\% de 
2000 à 2030. Les zones de stabilité ont diminué de 60,85\%. Ainsi, la dynamique de l'occupation du sol a généré des changements qui menacent plus les zones de stabilité. Cette situation induit la fragmentation du paysage à travers les changements constatés surtout de 2025 à 2030 sur certaines unités végétales comme la forêt dense humide et les savanes arborée et arbustive.

\section{Mesure de la fragmentation du paysage}

La conséquence des changements opérés sur les unités d'occupation du sol est la fragmentation du paysage d'étude. La figure 13 ci-dessous en présente les différentes valeurs.

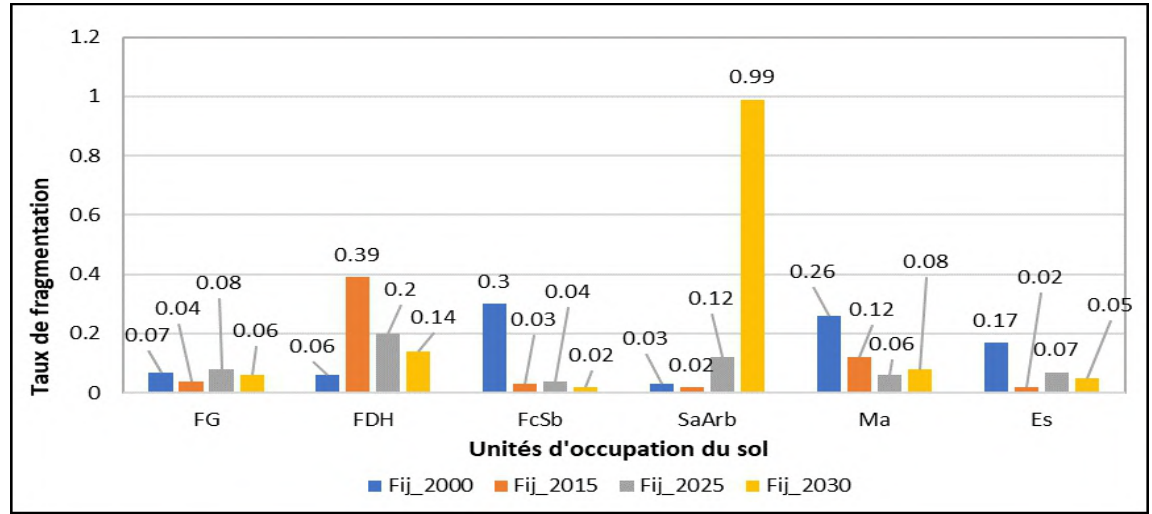

Figure 13 : Taux de fragmentation des formations végétales et humides Source : traitement statistique des résultats de cartographie, 2019

Légende : FG- Forêt- galerie ; FDH- Forêt dense humide- FcSb- Forêt claire et savane boisée ; Savanes arborée et arbustive ; Ma-Marécage ; Es-Eau de surface

L'analyse de la figure 13 montre que l'indice de fragmentation du paysage varie suivant les périodes d'étude et les formations naturelles considérées. En 2000, les valeurs les plus élevées de cet indice sont observées au niveau de la forêt claire et savane boisée $(0,30)$, des marécages $(0,26)$ et des eaux de surface $(0,17)$. Les valeurs les plus faibles de la fragmentation des unités d'occupation du sol pendant cette période caractérisent les savanes arborée et arbustive $(0,03)$, la forêt-galerie $(0,07)$ et la forêt dense humide $(0,06)$. En 2015, la fragmentation la plus importante est constatée sur la forêt dense humide $(0,39)$, et les plus faibles sur les autres formations végétales. En 2025, la forêt dense humide sera toujours la plus fragmentée, mais présentera un taux moindre $(0,20)$. En 2030 , on observera une fragmentation très importante des savanes arborée et arbustive $(0,99)$ et faible des marécages $(0,08)$ et des eaux de surface $(0,05)$.

Ainsi, de ce qui précède on peut dire que de 2000 à 2030, la forêt claire et savane boisée, les marécages et les eaux de surface vont connaître une fragmentation régressive alors que les savanes arborée et arbustive 
exprimeront une fragmentation progressive. En 2030, cette dernière unité sera fragmentée à $99 \%$ à cause des changements qui y seront observés.

\section{Discussion}

La présente étude a montré les possibilités qu'offre l'imagerie satellitaire pour la cartographie, la modélisation et l'analyse de changements de l'occupation du sol. Les résultats obtenus ont révélé que la dynamique spatio-temporelle de l'occupation du sol est caractérisée par la pression anthropique sans cesse croissante de l'agriculture et de l'urbanisation sur les formations naturelles. Cette situation exprime une intensité importante de changement pour les savanes arborée et arbustive et la forêt claire et savane boisée, quelle que soit la période d'étude. La conséquence des changements observés sur l'occupation du sol est la fragmentation progressive des savanes arborée et arbustive qui atteindra $99 \%$ en 2030. Selon certains auteurs (Mama et al., 2014 ; Ariori \& Ozer, 2005), les savanes d'Afrique connaissent actuellement des processus rapides de transformation des paysages ruraux et une dégradation des ressources naturelles. Pour Sounon Bouko et al. (2007), l'ouverture de nouveaux champs se fait au détriment des espaces forestiers et des savanes. Quant à Orékan (2014), les modifications de l'utilisation des sols dues à des facteurs anthropiques influent sur les futurs taux d'évapotranspiration et les précipitations régionales. Brun et al., 2018 ont montré que dans les zones humides, les facteurs de dégradation du paysage sont non seulement liés aux aléas climatiques, mais surtout aux activités anthropiques. Les résultats du présent travail confirment ceux des auteurs précités. Son intérêt réside dans la possibilité de révéler les vitesses des changements futurs de l'occupation du sol et les taux de conversion de ses unités les plus vulnérables afin d'assurer une bonne gouvernance territoriale des zones agricoles.

\section{Conclusion}

L'utilisation de l'imagerie satellitaire a permis de réaliser la cartographie diachronique, la modélisation et l'analyse des changements de l'occupation du sol du territoire de la Lama, sous - pôle du $7^{\text {ème }}$ pôle de développement agricole du Bénin. Les résultats obtenus montrent que de 2000 à 2030, la dynamique de l'occupation du sol génère des changements qui sont caractérisés par des taux de conversion différents pour chaque unité d'occupation du sol. Les savanes arborée et arbustive et la forêt claire et savane boisée sont particulièrement exposées. La cartographie des intensités de changement révèle des vitesses rapides, lentes et des zones de stabilité de la réalisation de cette conversion. La conséquence des changements dans la dynamique de l'occupation est la fragmentation du paysage qui est régressive pour la forêt claire et savane boisée, les marécages et les eaux de surface et 
progressive pour les savanes arborée et arbustive avec un taux particulièrement important de $99 \%$ en 2030. C'est pourquoi des dispositions urgentes doivent être prises pour protéger cette formation végétale afin d'assurer une bonne gouvernance territoriale et un équilibre écologique du paysage.

\section{References:}

1. Abdel-Kawy, O. R., Rod J. K., Ismail H. A., Suliman A. S., 2011, Land use and land cover change detection in the western Nile delta of Egypt using remote sensing data. Applied Geography, 31, 2, pp. 483-494.

2. Ariori, S.L. et Ozer, P., 2005, Évolution des ressources forestières en Afrique de l'Ouest soudano - sahélienne au cours des 50 dernières années. Geo-Eco-Trop, 29, pp.61-68.

3. Arouna, O., 2012, Cartographie et modélisation prédictive des changements spatio-temporels de la végétation dans la Commune de Djidja au Bénin : implications pour l'aménagement du territoire. Thèse de doctorat. Université d'Abomey-Calavi. Bénin. 162 p.

4. Bamba, I., 2010, Anthropisation et dynamique spatiotemporelle de paysages forestiers en République démocratique du Congo. Thèse de doctorat, Université Libre de Bruxelles, Belgique, 189 p.

5. Benkouider, F., Hamami, L., Abdellaoui, A., Salmon M., 2012, Extraction de routes par classification supervisée et par réseaux de neurones artificiels à partir d'image SPOT : cas d'une ville oasienne (Algérie), Revue Télédétection, Editions des Archives Contemporaines / Editions scientifiques GB / Gordon and Breach Scientific Publishers 11, 1, pp. 237-249.

6. Bogaert, J. \& Hong S.K., 2003, Landscape ecology: monitoring landscape dynamics using spatial pattern metrics. In: Ecological issues in a changing world, (eds. Hong, S.K.; Lee, J.A.; Ihm B.S.; Farina A.; Son Y.; Kim E.S. \& Choe J.C), Kluwer Academic Publishers, Dordrecht, pp. 109-131.

7. Bouko, B. S., Sinsin, B. \& Soulé, B. G., 2007, Effets de la dynamique d'occupation du sol sur la structure et la diversité floristique des forêts claires et savanes au Bénin, Tropicultura, 25, 4, p.221-227.

8. Brun, E.L., Djego, J. G., Gibigaye, M. et Tente, B., 2018, Dynamique De L'occupation Du Sol Dans Les Zones Humides De La Commune D'allada Au Sud-Benin (Sites Ramsar 1017 Et 1018), European Scientific Journal Edition, Vol.14, No.12, (Print) e - ISSN : 18577431 , pp.59- 77 .

9. Djohy, G. L.1, Totin Vodounon S. H., Kinzo N. E.1, Sinwongou M. A.1, Avahouin C. N. N.1, Akplogan K. N.1, Doumahoun D. S. E.1, 2017, Extrêmes climatiques dans le domaine soudanien au Bénin : 
étude comparée des perceptions populaires et des données climatologiques de l'ASCENA, Actes du XXXe colloque de l'association internationale de climatologie: Climat, ville et environnement, Tunis, pp. 281-550

10. FAO, Bénin, 2010, Rapport national, Bénin, FRA2010/022 Rome, 54

11. FAO, Rome, 2015, natural resources, FAO/World Water Council, ISBN $978925107877874 \mathrm{pp}$.

12. Hibbarda ,K., A. Janetos, D.P. van Vuuren, J. Pongratz, S.K. Rose, R. Betts, M. Herold et J.J. Feddema, 2010, Research priorities in land use and land-cover change for the Earth system and integrated assessment modelling. International Journal of Climatology, 30, 13, pp. 21182128.

13. INSAE, 2016, Effectifs de la population des villages et quartiers de ville du Bénin (RGPH-4, 2013), 83p.

14. Jensen, J.R., 1996. Introductory digital image processing: a remote sensing perspective, 2nd ed., Prentice Hall, Upper Saddle River, New Jersey, 316p.

15. LA FAO ET LES ODD, 2017, [En ligne] URL : www.fao.org

16. Lawrence, P.J. and Chase T.N., 2010, Investigating the climate impacts of global land cover change in the Community Climate System Model (CCSM). International Journal of Climatology, 30, pp. 2066 2087.

17. Mairie de Porto-Novo, 2006, Monographie complète des communes des départements de l'atlantique et du littoral, Rapport d'étude, $28 \mathrm{p}$.

18. Mama, A., Sinsin, B., De Cannière, C., Bogaert, J., 2014, Anthropisation et dynamisation des paysages en zone soudanienne au nord du Bénin. Tropicultura, 31,1, pp. 78-88.

19. Ministère de l'Environnement, de l'Habitat et de l'Urbanisme, Direction Générale de l'Environnement, UNDP- FEM, 2011, Deuxième communication nationale de la république du bénin sur les changements climatiques, Rapport d'étude, 165p.

20. Ministère du Cadre de Vie et du Développement Durable (MCVDD), 2017, Programme de définitions des cibles nationales de la Neutralité de dégradation des Terres (PDC/NDT), Benin LDN TSP, Rapport d'étude sur le Bénin.

21. Monmonier, M., 1974, Measures of Pattern Complexity for Choropleth Maps, Cartography and Geographic Information Sciences, 1,2, pp.159-169.

22. Mugisha, S., Tenywa, M. M., \& Burt, P. J. A., 2010. An improved technique for the prediction of optimal image resolution(s) for largescale mapping of savannah ecosystems. African Journal of Environmental Science and Technology, 4 (10): 709-717. 
23. Oloukoi J., Mama V. J., et Agbo B. F., 2006, Modélisation de la dynamique de l'occupation des terres dans le département des collines au Bénin, Revue Télédétection, vol. 6, n 4, p. 305-323

24. Oloukoi, J., 2013, Scénario socio-économique et écologique des changements de l'occupation des terres au Bénin, [VertigO] La revue électronique en sciences de l'environnement, Volume 13, Number 1, pp. 2-22.

25. Orékan, V., Abdoulaye, D., Houndagba, C. J, Sinsin, B., 2014, Dynamique prospective des écosystèmes forestiers classés au Bénin : quelle perspective pour une utilisation durable. [En ligne] URL : http://www.bj.chmcbd.net > RESSOURCES > Publications > Flore $>173$.

26. Pontius, R. G., 2000, Quantification error versus location in comparison of categorical maps. Photogrammetric Engineering and RemoteSensing, 66, 8), pp.1011-1016.

27. Présidence de la République du Bénin, «Programme d'Action du Gouvernement 2016-2021 », version synthèse, pp. 1-28.

28. Sambiéni, K.R., Toyi, M. S. et Mama, A., 2015, Perception paysanne sur la fragmentation du paysage de la Forêt classée de l'Ouémé Supérieur au nord du Bénin, [VertigO] La revue électronique en sciences de l'environnement, volume 15, numéro 12, pp.1-18.

29. Shiferaw, A., 2011, Evaluating the land use and land cover dynamics in Borena Woreda of South Wollo highlands, Ethiopia. Journal of Sustainable Development in Africa, 13, 1, pp. 87-107.

30. Singh, A., 1989, Review Article Digital change detection techniques using remotely-sensed data, International Journal of Remote Sensing, 10:6, pp. 989-1003

31. Takahashi, H.G., Yoshikane, T., Hara, M., Takata, K., Yasunari, T., 2010, High-resolution modelling of thepotential impact of landsurface conditions on regional climate over Indochina associated with thediurnal precipitation cycle, International Journal of Climatology, 30, pp. $2004-2020$.

32. Toko Mouhamadou, I., 2014, Facteurs déterminants de la fragmentation des écosystèmes forestiers : cas des îlots de forêts denses sèches de la forêt classée des Monts Kouffé et de sa périphérie au Bénin, Thèse de Doctorat Unique Université d'Abomey-Calavi, Gestion de l'Environnement, $\mathrm{N}^{\circ}$ d'Enregistrement : $\mathrm{N}^{\circ}$ 23414/EDP/FLASH/UAC, 235 p. 